\title{
Design and Development of Photography and Videography Simulation System Facing Multiple Platforms
}

\author{
Dan Zhao ${ }^{1, \mathrm{a}}$, Hui-jie $\mathrm{Qu}^{1, \mathrm{~b}}$, Yue-fei $\mathrm{Gao}^{2, \mathrm{c}}$ and Xing $\mathrm{Li}^{1, \mathrm{~d},{ }^{*}}$ \\ ${ }^{1}$ School of Mathematics and Information Science, Guangxi College of Education, Nanning 530023, \\ China \\ ${ }^{2}$ Educational Technology and Information Management Center, Guangxi College of Education, \\ Nanning 530023, China \\ azhaodan0519@163.com, bqq_rr252@126.com, '850506135@qq.com, \\ ${ }^{\mathrm{d}}$ GXEC_lixing@foxmail.com \\ * Corresponding author: Xing LI
}

Keywords: Photography and videography, Virtual reality, Simulation, Multiple platforms.

\begin{abstract}
Through analyzing present situations of photography and videography teaching, according to problems like expensive equipment damage caused by unfamiliarity in photography and videography teaching as well as lack of professional equipment, the author narrated necessity of applying the simulation system in teaching practice. The system adopted technologies like databases and Android, and integrated mobile training, digital training and interactive training to solve data resource support in multiple platforms. It eliminated restrictions to courses caused by Internet and equipment and had much interaction in class. It also improved quality of classes, reduced damage to equipment, and solved the problem of lack of equipment. Thus, it had significant value in use.
\end{abstract}

\section{Introduction}

The technology of virtual reality is also called "immersive multimedia" or "computer simulation of reality". It is regarded as one of the important developing subjects and one of the important technologies that influence people's life in the 21st century. It is a new technology that integrates results in many fields, like computer graphics, the technology of human-computer interface, the technology of sensor and the technology of artificial intelligence. The aim is to improve functions of human-computer interaction, and get real visual, touching, auditory and smelling experience results (Yang et al.2010). Through visualized expression of data and analyses of human-computer interaction, virtual learning environment can increase users' sense of immersion in computer virtual of reality. In 1980s, the technology of virtual reality began to be applied in industries other than entertainment, especially professional education and training. For example, the technology of virtual reality was used to train pilots. In 1990s, American researchers introduced the technology of virtual reality into basic education and higher education in Science Space. Research projects included Cell Biology, Global Change, Virtual Gorilla Exhibit, etc. China also highly emphasized application of the technology of virtual reality in education. By 2016, Ministry of Education had approved 300 state-level virtual simulation experimenting and teaching centers (Ministry of Education, 2016). Recently, Science popularization Department of China Association for Science \& Technology publicized construction of 35 virtual reality science and technology museums (China Association for Science \& Technology, 2016) [1].

\section{Analyses of Present Situation of Photography and Videography Courses}

In recent years, as entertainment keeps developing, the major of photography and videography technologies has been set in many universities and colleges and become a popular major. Thus, the major of photography and videography has been paid more and more attention. It can effectively connect photography in society and cultivate a series of talents with practical skills. In traditional 
teaching of photography and videography, problems mainly include disconnection of theories and practice, lack of professional equipment, expensive equipment damage caused by unfamiliarity and extremely high requirement for teachers.

As there is progress in science and technology, teaching modes of photography and videography have changed. Firstly, there occurred digital teaching that makes up for restrictions of time and place for traditional teaching and the disadvantage of high costs in building on-site practice base. However, traditional digital teaching has limited content and lacks intelligence. Two-dimensional webpages cannot realize dynamic interaction between real environment and participants and its teaching results are not satisfying. Therefore, the technology of virtual reality is introduced on the basis of digital teaching. Combination of the technology of virtual reality and digital teaching generates three -dimensional webpages, and uses human behaviors as the main body of the browser. All expressions change as behaviors of operators change. It changes disadvantages of bad interaction of former webpages. But this kind of training is mostly based on Local Area Network and has many restrictions for visited IP. It cannot assure that students can visit the system to study in any place at any time.

According to above problems, the author combined the server technology of ASP. NET and the technology of virtual reality, adopted the cross-platform development tool of Unity3D and the popular tool of Visual Studio, and used C\# to develop the virtual simulation system that supports mobile phones, tablet computers and PCs in online and offline modes for students to selectively study in various terminals according to their own conditions.

\section{Framework and Design Procedure of System}

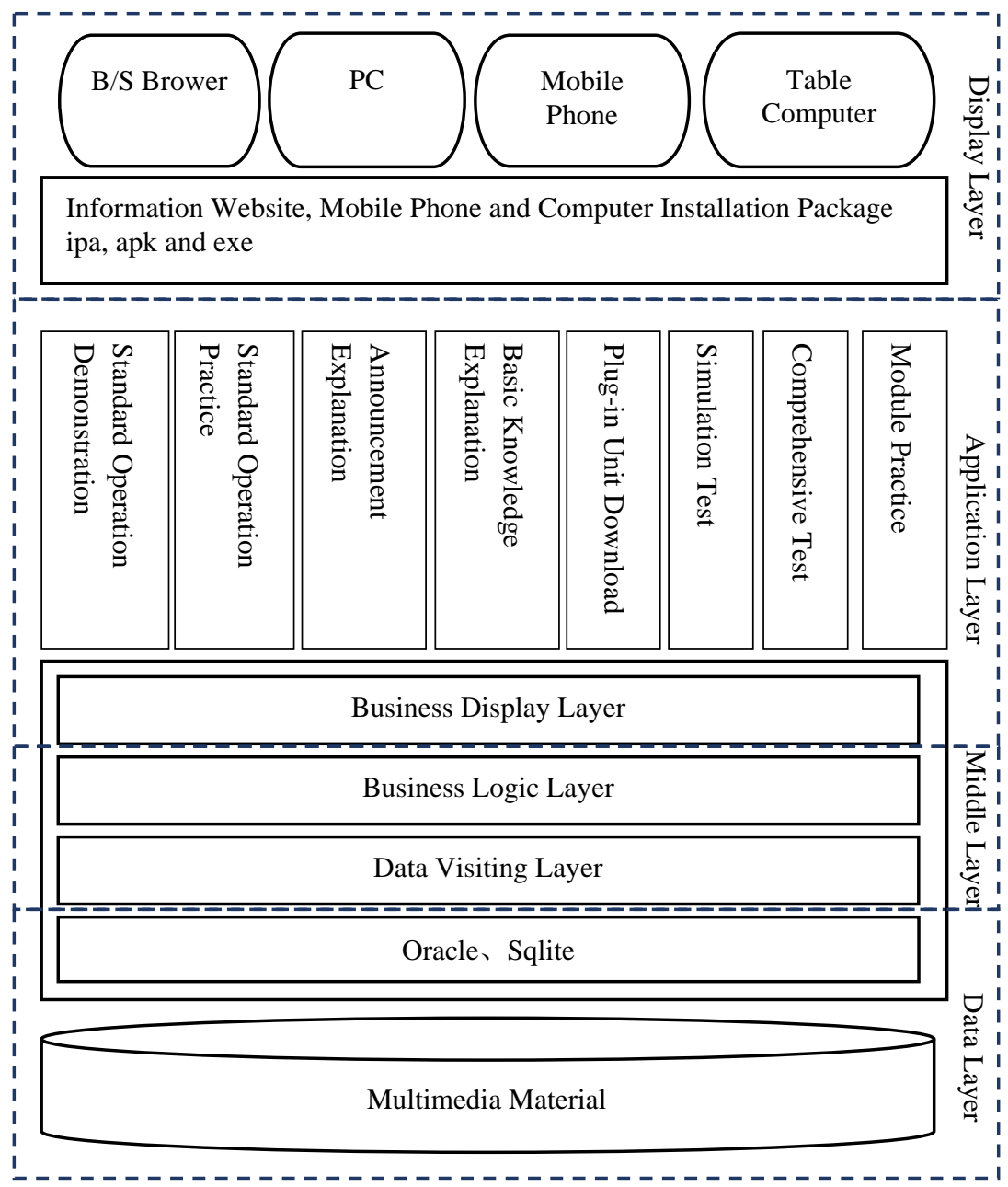

Fig.1 Framework of System 
The photography and videography simulation system facing multiple platforms adopted the four-layer system structure of B/S+C/S and supported mobile phones, PCs and tablet computers. The framework of system is shown in Fig.1.There were mainly three aims to develop this system: the first aim was to enable learners to experience the virtual videography experiment, the dynamic and vivid virtual learning mode developed by the technology of virtual reality; the second aim was to enable learners to command the most basic and crucial photography and videography skills through the virtual videography experiment (for example, adjustment of white balance, adjustment of the focal distance, coordinated use of the diaphragm and the shutter, photography of fixed scenes, photography of moving scenes, composition practice and photography review practice, etc.); the third aim was to solve the problem that students in school or of long-distance education cannot attend experiment classes.

Through analyzing characteristics of students' electronic equipment, this system could finally generate the pc version, webpage version and mobile phone version. The system development procedure is shown in Figure 2.

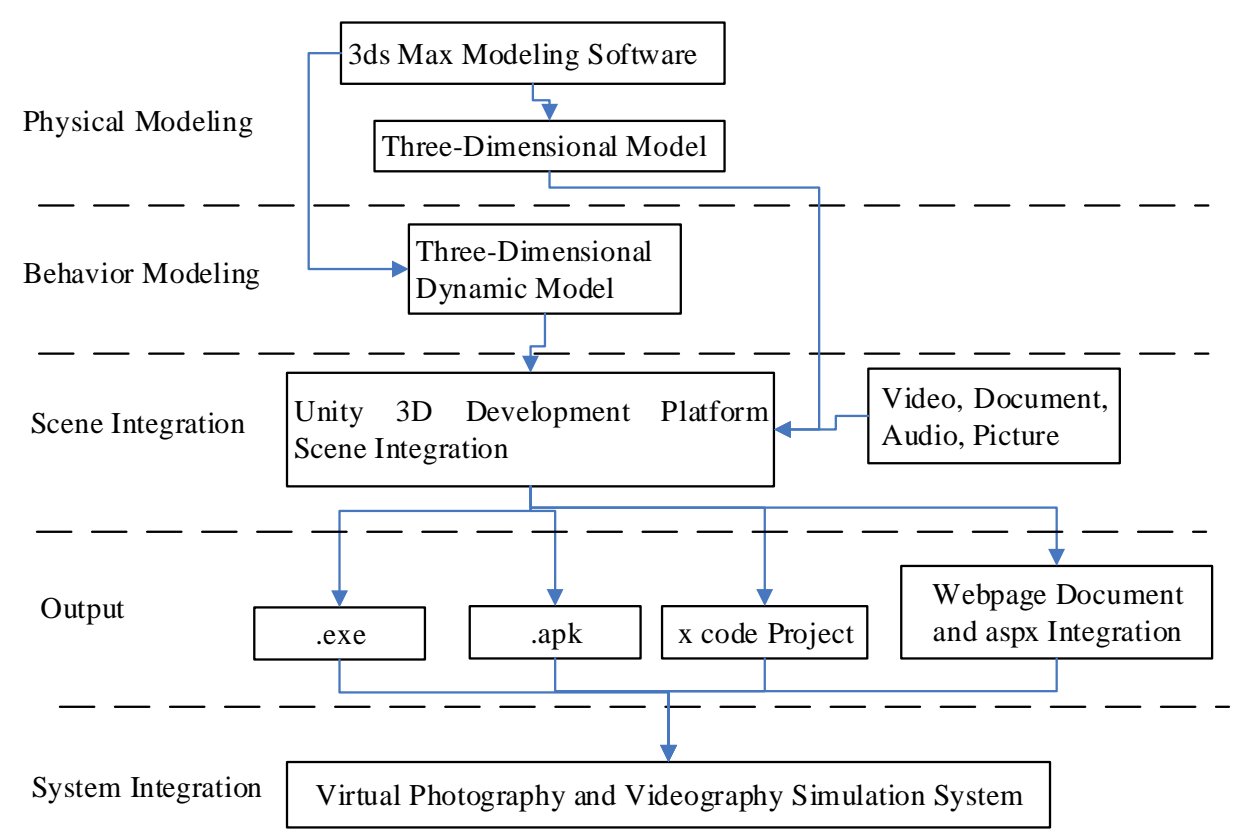

Fig.2 System Development Procedure

\section{Key Problems}

\section{Learning Mode Design}

(1)Standard operation demonstration mode

It was the demonstration mode of safe operation. The system demonstrated strictly according to operation rules, and did not respond to operation that did not conform to operation rules. The main purpose was to enable new trainees to get familiar with correct operation rules of new tasks through correct demonstration.

(2)Guide operation mode

It was the training mode for safe operation according to tips of the guide. At the beginning of every step, the tip was given in simple words to indicate how to operate. Trainees operate relevant equipment and parts using input equipment.

(3)Open free operation mode

It was the "real" training mode for safe operation. It could make sure that trainees could operate all parts (correctly and wrongly) in the revolved range. The system simulated effects of the real world according to relevant knowledge, and expressed them in the virtual environment. 
The open free operation mode was the closest to operation behaviors and responding modes of participants in the real world. It allowed participants to operate wrongly. The system responded and gave tips according to knowledge in the field (including occurrence of accidents), which was much beneficial for trainees to have deeper impression and improve operation level.

\section{Multiple Date Sources}

The system in this paper could be applied in different platforms, while different platforms supported different data sources. Thus, the system provided support for many data sources. The mobile phone version and the EXE version adopted the database of Sqllite; the website version adopted the database of oracle. Besides, the upload button and the download button were used to ensure consistency between data of clients and those of servers.

(1)Visit of Databases on Different Platforms

This system adopted the database of Sqllite. It needed to judge the platform for connection. By default, it was operated on computer and codes of its connection with the database were:

string path = Application.dataPath +"/Plugins/windows/assets/" + "orcl1.db";

$\mathrm{db}=$ new DbAccess ("URI=file:" + path);

\#if UNITY_EDITOR was used to judge whether it was operated on Android. If it was, codes of its connection with the database were:

String appDBPath = Application.dataPath +"/Plugins/Android/assets/" + "orcl1.db";

$\mathrm{db}=$ new DbAccess ("URI=file:" + appDBPath);

(2)Visit of Oracle

The database that the website version used was Oracle. When visiting Oracle, catenation strings of the database were put in Web.config. The method of AppSettings in ConfigurationManager in the program was used to acquire catenation strings of the database. After acquiring them, connection with oracle was created and codes are:

Connection = new OracleConnection(ConnectionString);

Connection.Open();

\section{Control Screen Self-Adaptation}

Since there were many kinds of electronic equipment with different screen sizes and resolutions in markets at present, the problem of screen resolution self-adaptation on different equipment in developing the virtual simulation system. In this paper, the author researched the problem of screen resolution self-adaptation of the photography and videography simulation system developed for Unity3D on different mobile equipment

Application based on Unity3D would make normal UI in a mess on terminals with different screen resolutions [2,3]. Experiments proved that difference in resolution would result in deviations of place and size for UI. In the circumstance where the screen size was the same, dots per inch was different. The larger DPI (dots per inch, dots was the minimum display unit for screens) was, the larger the quantity of dots in the same area was. Thus, on screens of different DPI, controls of the same pixel were displayed differently. The larger the density was, the more dots were in the same area and the smaller dots were. Thus, the same object would be displayed smaller on the screen of larger density. To solve this problem, it was necessary to carry out conversion between DP (Density-independent-pixel, abstract pixel) and PX (pixel, the basic unit of image display):

$$
\mathrm{PX}=\mathrm{DP} *(\mathrm{DPI} / 160)
$$

From this it is obvious that for the screen of 160DPI, 1DP = 1PX. If DP is used as the unit to define the size and the place of UI, consistent effects will be displayed on screens of different DPI, so as to realize irrelevance of DPI.

In order to adapt the system based on Unity3D to the resolution of equipment, it was necessary to consider two aspects, namely, aspect ratio and view size [4]. According to statistics, from Table 1 it is obvious that aspect ratios of most equipment were close to 1.7. If UI was made according to this 
aspect ratio on the screen with the resolution of $1024 \times 600$, displays on different mobile equipment would not have too long distances between each other longitudinally or crosswise. If aspect ratios were to be adjusted the same, firstly it was necessary to define a standard screen resolution SW = 1024f, $\mathrm{SH}=600 \mathrm{f}$. Then, width $\mathrm{W}$ and height $\mathrm{H}$ of the used equipment should be acquired. Define zooming coefficient formula sfW $=\mathrm{W} / \mathrm{SW}, \mathrm{sfH}=\mathrm{H} / \mathrm{SH}$. Experiments should be carried out on screens with the resolution of $800 \times 480$ and $1280 \times 800$. Relative places and sizes of objects should be displaced and zoomed in the equal ratio according to screen resolutions.

Table 1 Aspect Ratio of Screens of Mobile Equipment

\begin{tabular}{|c|c|c|}
\hline Height & Width & Aspect Ratio \\
\hline 800 & 480 & 1.6667 \\
\hline 1280 & 720 & 1.7778 \\
\hline 960 & 540 & 1.7778 \\
\hline 1280 & 800 & 1.6 \\
\hline 960 & 640 & 1.5 \\
\hline 1184 & 720 & 1.6444 \\
\hline 1920 & 1080 & 1.7778 \\
\hline
\end{tabular}

\section{Conclusion}

As the media era has come, photography and videography technologies are playing a more and more important role in teaching. Traditional teaching modes gradually express many disadvantages. They emphasize theories but ignore practice. Since equipment is expensive, there is a lack of hardware. And due to unfamiliarity of students with equipment, equipment is easily damaged in operation. In order to solve these problems, the author used 3dsmax and Unity3D as development platforms to develop a simulation system that combined digital training, interactive training and mobile training, eliminating restrictions of time and place for students to learn and enabling them to learn more efficiently in any place at any time. Due to immersion, interaction and imagination of virtual reality, students can learn vividly on multiple platforms like mobile phones, PCs and table computers. It solved the problem of equipment operation due to costs, and eliminated restrictions of Internet and equipment for courses. Since students have practiced on the virtual simulation system and got familiar with operation modes of equipment, damage of equipment is largely reduced. Thus, this system has important application significance in use.

\section{Acknowledgment}

This research was financially supported by the Education Department of Guangxi Province(NO.2 015JGZ172), and scientific research projects of Guangxi College of Education in 2015 (B2015004, No. YB2014587, NO.A2015002), and 2015 Special Program on Hybrid Teaching Reform for Education and Teaching Reform of Guangxi College of Educati on (NO.XJJG15A03), and 2016 Programng Teams with "Key Competencies” in Guangxi College of Education, Topic: Research on Integrating Cultivation of Key Competencies into Teaching

\section{References}

[1] De-Jian LIU. Potential of Virtual Reality Technology in Education _ Progress and Challenges. Open Education Research, 2016.

[2] Lin-Xuan LV. “All People Run” Client Design and Realization Based on Unity3D. Beijing: Beijing Jiaotong University, 2016.

[3] Zhao Y, Yan C, Zhou X, et al. The research and development of 3D urban geographic information system with Unity3D. International Conference on Geoinformatics,2013. 
[4] Jing-Ming XIE. Research on Key Technologies Base Unity3D Game Engine. The 7th International Conference on Computer Science \& Education(ICCSE2012),2012.

[5] Jian-Song QIU. Research and Realization of Real-Time Virtual Simulation System. Practical Electronics, 12 (2012)11-12.

[6] Hui LIU. Research on Application Scenes of VR Technology. Electronic Commerce, 2017

[7] Yu-Bin CHEN, Yu-Zhu ZENG. Web3D Display Based on Unity3D. Computer Programming Skills \& Maintenance, 2012 